\title{
Screen-Printed Flexible Bandstop Filter on Polyethylene Terephthalate Substrate Based on Ag Nanoparticles
}

\author{
Rajendra Dhakal, ${ }^{1}$ Younsu Jung, ${ }^{2}$ Hyejin Park, ${ }^{2}$ Gyoujin Cho, ${ }^{2}$ and Nam Young Kim ${ }^{1}$ \\ ${ }^{1}$ Department of Electronic Engineering, Kwangwoon University, Nowon-gu, Seoul 139-701, Republic of Korea \\ ${ }^{2}$ Department of Printed Electronics and Chemical Engineering, Sunchon National University, Sunchon, \\ Jeonnam 540-742, Republic of Korea \\ Correspondence should be addressed to Nam Young Kim; nykim@kw.ac.kr
}

Received 19 June 2015; Revised 4 September 2015; Accepted 15 September 2015

Academic Editor: Bhupendra K. Sharma

Copyright (c) 2015 Rajendra Dhakal et al. This is an open access article distributed under the Creative Commons Attribution License, which permits unrestricted use, distribution, and reproduction in any medium, provided the original work is properly cited.

We present a low-power, cost-effective, highly reproducible, and disposable bandstop filter by employing high-throughput screenprinting technology. We apply large-scale printing strategies using silver-nanoparticle-based ink for the metallization of conductive wires to fabricate a bandstop filter on a polyethylene terephthalate (PET) substrate. The filter exhibits an attenuation pole at $4.35 \mathrm{GHz}$ with excellent in-and-out band characteristics. These characteristics reflect a rejection depth that is better than $-25 \mathrm{~dB}$ with a return loss of $-0.75 \mathrm{~dB}$ at the normal orientation of the PET substrate. In addition, the filter characteristics are observed at various bending angles $\left(0^{\circ}, 10^{\circ}\right.$, and $\left.20^{\circ}\right)$ of the PET substrate with an excellent relative standard deviation of less than $0.5 \%$. These results confirm the accuracy, reproducibility, and independence of the resonance frequency. This screen-printing technology for welldefined nanostructures is more favorable than other complex photolithographic processes because it overcomes signal losses due to uneven surface distributions and thereby reveals a homogeneous distribution. Moreover, the proposed methodology enables incremental steps in the process of producing highly flexible and cost-effective printed-electronic radio devices.

\section{Introduction}

Flexible cost-effective devices, such as radiofrequency identification (RFID), antennas, and filters, are needed for enabling realization of high-performance devices for operation in frequency bands of the monolithic microwave integrated circuit (MMIC) and radiofrequency integrated circuit (RFIC). Recently, more sophisticated processes have been used for printing various types of electronic devices and systems. These printing processes are critical for replacing the more complicated, time-consuming, and expensive photolithographic process. One of the most powerful device fabrication methods, photolithography, is widely used for miniaturized microwave devices composed of metallic lines of only a few microns wide. However, photolithography is a rather complicated process, even for creating a few samples with simple structural designs $[1,2]$.
The screen-printing technique produces electronics devices on paper as well as on plastic foil with different thicknesses and dielectric constants, which can be tailored to design requirements [3]. High-performance, low-cost, and flexible narrowband bandstop (NBBS) filters with a minimum size and weight, high-frequency selectivity, and excellent pass-band insertion and return losses are important for C-band applications in modern communication systems. These parameters are the key factors for the development of demanding, miniature, and multifunctional communication systems for wireless sensor networks and smart electronic devices. The deposition of suitable metal ink on the prescribed flexible substrate for the fabrication of highly desirable electronic devices is a major challenge $[4,5]$. This metallization itself presents the challenge of the formulation of suitable silver $(\mathrm{Ag})$ nanoparticle ink. The metal precursor ink must provide a sufficiently high resolution and good 
adhesion to the surface with good morphological structure within the Ag nanoparticles. Accordingly, signal loss due to the skin effect can be easily studied and minimized $[6,7]$.

However, no technology exists that is sufficiently advanced for building a flexible, high-throughput, costeffective, and highly reproducible bandstop filter (BSF). The design of flexible single-band BSF on a PET substrate with high stopband attenuation and low return loss has not yet been reported. The imprecise formulation of the Ag-nanoparticle-based ink has resulted in design complexity for uniform width and thickness of the transmission-line. Furthermore, it has degraded the results in the return loss and rejection depth [8]. Various processes and strategies have been previously proposed for bandstop filter design. These studies have focused on fabrication using flame retardant 4 (FR4), low-temperature cofired ceramic (LTCC), coplanar waveguide, and metamaterials, with complex and expensive photolithography being the most common method of fabrication [9-14]. Screen-printing is a promising approach for large-scale fabrication with minimal radial loss by the skin effect at high frequency with even surface characterization electroplated by $\mathrm{Ag}$ nanoparticles. This approach has surpassed the aforementioned complex photolithography process.

To address the above limitations, we propose a simple and cost-effective method to manufacture a highly reproducible BSF on a PET substrate by employing high-throughput screen-printing technology with an attenuation pole at 4.35 GHz. Excellent performance of transmission parameters in the stop band with a good morphological characterization of the Ag nanoparticles justifies the operational characteristics of BSF. Through experiments, we demonstrate the relative standard deviation (RSD) for five consecutive experiments in each bending angle of the substrate. The result of an RSD value of less than $0.5 \%$ confirms the accuracy, reproducibility, and independence of the resonance frequency despite the flexibility of the substrate.

\section{Device Fabrication}

2.1. Schematic Representation. The schematic structure of the proposed BSF was realized on a PET substrate. The Ag nanoparticles applied for the metallization of the conductive lines served as a signal carrier at high frequency. The concept of folded signal lines toward the input was implemented with capacitance in between the C-shaped stubs. The connection of folded meander-line with multiple bends can generate a specific resonance frequency. However, the rejection depth degrades and the topology will be too complex to bend the signal line. Furthermore, the complexity of the junction discontinuity effects will increase, such that an accurate value of the return loss cannot be obtained $[15,16]$. The proposed structure was therefore designed on a PET substrate with a thickness of $180 \mu \mathrm{m}$, a dielectric constant of 3.0, and a loss tangent of 2.5. Screen-printing technology was introduced along with Ag nanoparticles for the metallization of the BSF. To minimize the effective area of the filter, the length of the high-impedance section of the resonator was modified by

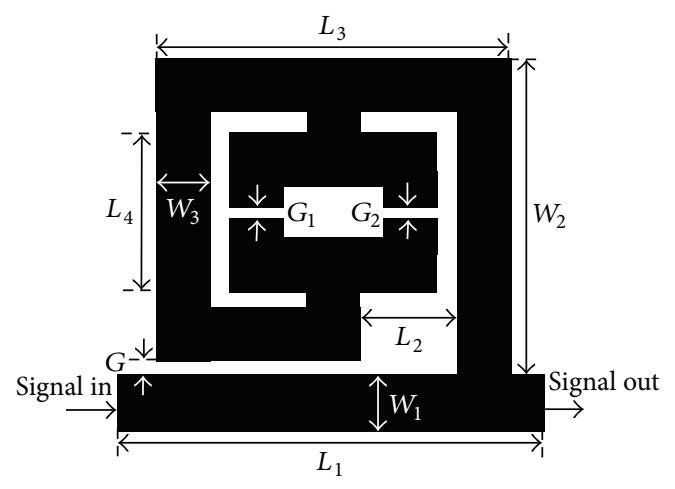

FIGURE 1: Schematic diagram showing the top view of the proposed NBBS filter.

bending it along the width. The filter can be used to suppress the resonance frequency $\left(f_{0}\right)$ by $-25.88 \mathrm{~dB}$ in the stopbands and simultaneously achieve a narrowband bandwidth with a $-10 \mathrm{~dB}$ fractional bandwidth (FBW) of $6.44 \%$. The layout of the proposed filter with detailed dimensions is $L_{1}=4 \mathrm{~mm}$, $L_{2}=0.91 \mathrm{~mm}, L_{3}=3.32 \mathrm{~mm}, L_{4}=1.49 \mathrm{~mm}, W_{1}=$ $0.53 \mathrm{~mm}, W_{2}=2.95 \mathrm{~mm}, W_{3}=0.5 \mathrm{~mm}, G=0.12 \mathrm{~mm}$, $G_{1}=0.10 \mathrm{~mm}$, and $G_{2}=0.10 \mathrm{~mm}$, as shown in Figure 1 .

2.2. Conductive Ag Metal Ink and Plastic Substrate. The use of $\mathrm{Ag}$ for metallization on the polymer PET substrate has gained considerable research interest. The deposition of $\mathrm{Ag}$ nanoparticles during metallization has been deemed favorable over other metals on account of its unique properties, such as chemical inertness, excellent electrical conductivity, and ease of ink formulation. A PET film (width of $200 \mathrm{~mm}$ and thickness of $180 \mu \mathrm{m}$, purchased from SKC, Korea) was used along with Ag-nanoparticle-based conducting ink (PG007 BB type, Paru Co., Korea). The silver ink was further formulated to meet the viscosity of $500 \mathrm{cp}$ and surface tension of $47 \mathrm{mN} / \mathrm{m}$ using ethylene glycol (Sigma-Aldrich) and dipropylene glycol methyl ether (Sigma-Aldrich), respectively.

2.3. Fabrication Process and Experimental Setup. For fabrication of the screen-printed bandstop filter, we used a single-step printing process through a screen printer (Sun Mechanix Co., Korea). With its ease of use for designing flexible devices, screen-printing is a remarkable technique, especially for printing on flexible substrate; moreover, the process is relatively simple, cost-effective, and versatile [17, 18]. The screen plate above the substrate was used to make the pattern. The screen plate was made with a stretchy fine lattice structure of 200 mesh. Ag ink was placed on top of the screen with a squeegee bar to fill it with ink, as shown in Figures 2(a) and 2(b). The movement of the squeegee with pressure enabled the Ag ink to pass through the pattern to create the Ag pattern designed on the PET substrate. The $\mathrm{Ag}$ ink was printed on PET film with a printing speed of $5 \mathrm{~mm} / \mathrm{s}$ and a pressure of $5 \mathrm{~kg}_{\mathrm{f}}$. The resulting $\mathrm{Ag}$ printed films were dried at $150^{\circ} \mathrm{C}$ for $10 \mathrm{~min}$. To provide the ground for the signal line of the bandstop filter, the Ag ink was 


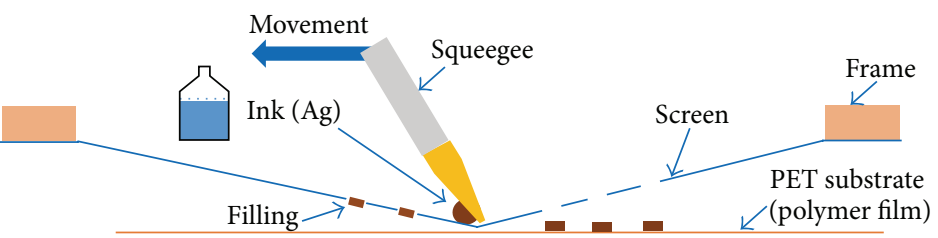

(a)

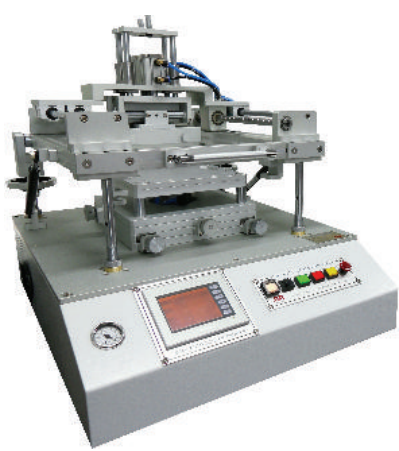

(b)

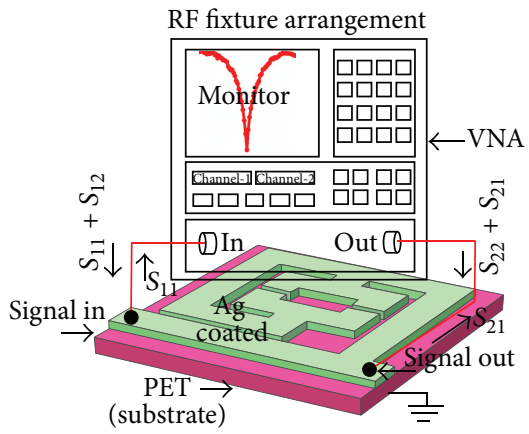

(c)

FIGURE 2: Schematic of (a) screen-printing process on polymer film, (b) semiautomatic screen printer, and (c) 3D representation as BSF on PET substrate connected to VNA.

reprinted on the backside of the PET substrate with a printing speed of $5 \mathrm{~mm} / \mathrm{s}$ and a pressure of $5 \mathrm{~kg}_{\mathrm{f}}$. It was additionally treated at $150^{\circ} \mathrm{C}$ for $10 \mathrm{~min}$, as represented in supplementary Figure S1 in Supplementary Material available online at http://dx.doi.org/10.1155/2015/978562. For the final testing of the BSF, it was encapsulated in printed circuit board (PCB) with a $50 \Omega$ port connected to the input and output sides of the transmission-line. The necessary transmission parameters were then obtained by connecting the input and output ports to an Agilent 8510C vector network analyser (VNA), as shown in Figure 2(c).

\section{Filter Design and Concept}

The design of the filter was implemented by the concept of the folded meander-line structure to generate the BSF with an attenuation pole at $4.35 \mathrm{GHz}$ with good selectivity, as demonstrated in Figure 3(a). The C-stub along the meander-line was folded to generate the stopband characteristics. Each bend of the meander-line represents a series inductor connected to a parallel capacitor for generation of the stopband characteristics of the proposed filter. The tank circuits $L_{2}, C_{2}$ and $L_{3}, C_{3}$ represent the equivalent inductance and capacitance for the bends $(1,2)$ and $(3,4)$, respectively, as represented in Figure 3(b). Figure 3(c) depicts a magnified view of the gap between the C-shaped stubs with gap capacitance $\left(C_{1 G 1}, C_{1 G 2}\right)$. The equivalent circuit representation of the transmission-line on the input and output sides was modeled by the inductor $\left(L_{t}\right)$ and capacitor
$\left(C_{t}\right)$, as represented in Figure $3(\mathrm{~d}) . Z_{\text {in }}$ and $Z_{\text {out }}$ represent the impedances of the transmission-line and are assumed to be $50 \Omega$. The distance between the C-stubs could be varied to generate variation in gap capacitance and ultimately the resonance frequency of the filter during the design analysis. The rejection depth is reflected by $S_{21}$, while $S_{11}$ generate the return loss for the BSF, as mentioned in Figure 3(d) $[19,20]$.

\section{Result and Discussion}

4.1. Structural and Compositional Analysis. The samples on PET substrates were characterized using X-ray diffraction (XRD), atomic force microscopy (AFM), and scanning electron microscopy (SEM). The surface characterization of the filter with respect to XRD and AFM was presented. A typical XRD pattern of the PET film is illustrated in Figure 4, where different peaks are assigned to represent the different structures and compositions of the PET film and Ag nanoparticles. For the tested PET film, a peak is observed at $2 \theta=25.87^{\circ}$ and at $38.13^{\circ}$, corresponding to $\mathrm{Ag}$ (111), which reveals the preferentially oriented growth of the Ag nanoparticles. The data obtained from the XRD measurement indicate the highquality Ag metallization on the PET substrate as a promising candidate for the application to flexible radio devices.

In addition, three peaks are observed for the $2 \theta^{\circ}$ angle, which correspond with planes (111), (200), and (220), with the highest diffraction intensity being for plane (111). Additionally, diffraction with lower intensity peaks is observed at a $2 \theta^{\circ}$ angle of $44.34^{\circ}$ (200) and $64.49^{\circ}$ (220) 


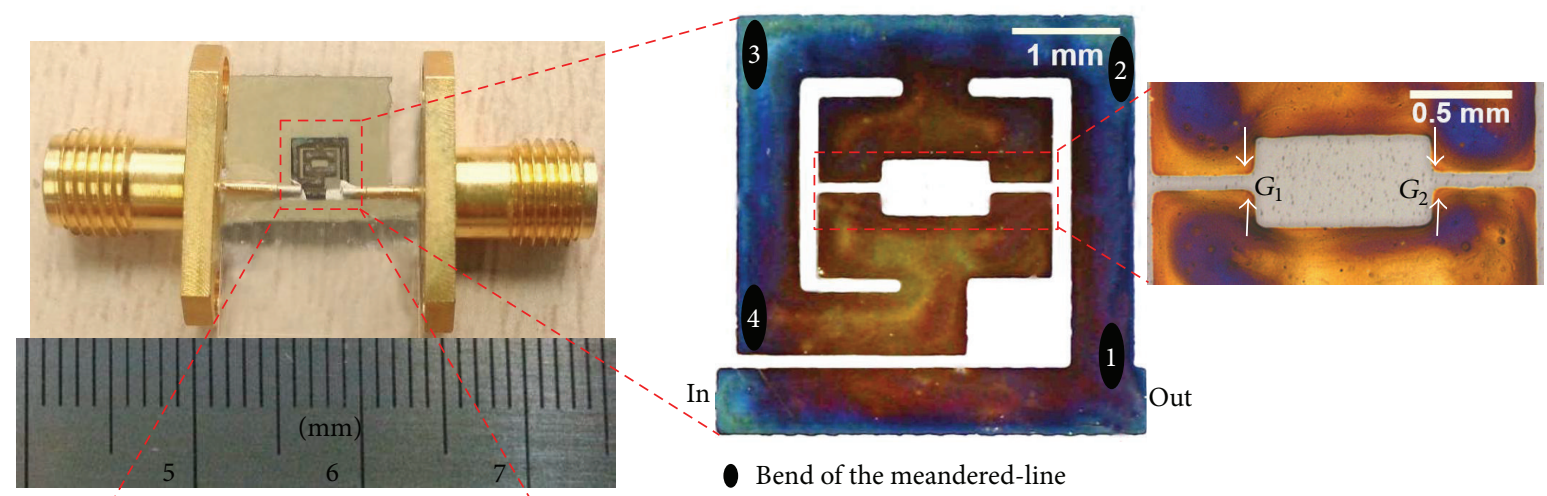

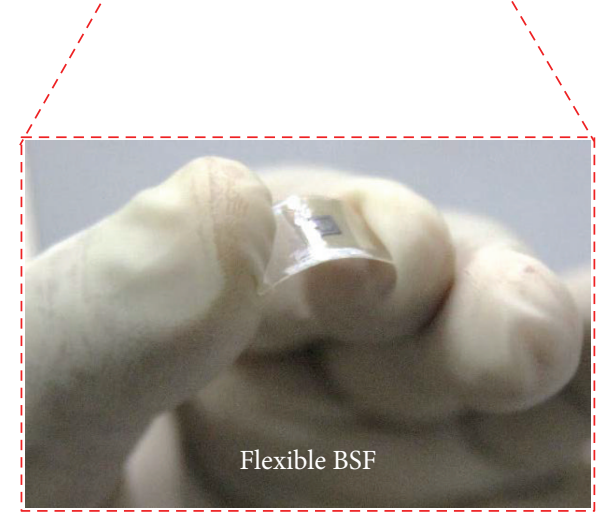

(a) (b)

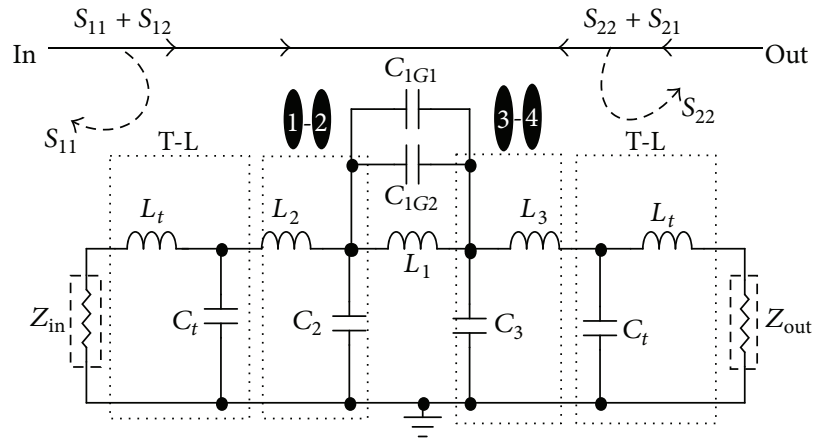

$\mathrm{T}-\mathrm{L}$ represent the transmission-line (c)

(d)

FIGURE 3: Illustration of the (a) fabricated flexible BSF, (b) optical microscopic view of the printed single layer device, (c) magnified view of the printed portion containing the gap capacitance $\left(C_{1 G 1}\right.$ and $\left.C_{1 G 2}\right)$, and (d) equivalent circuit model representing the behavior of the screen-printed BSF.

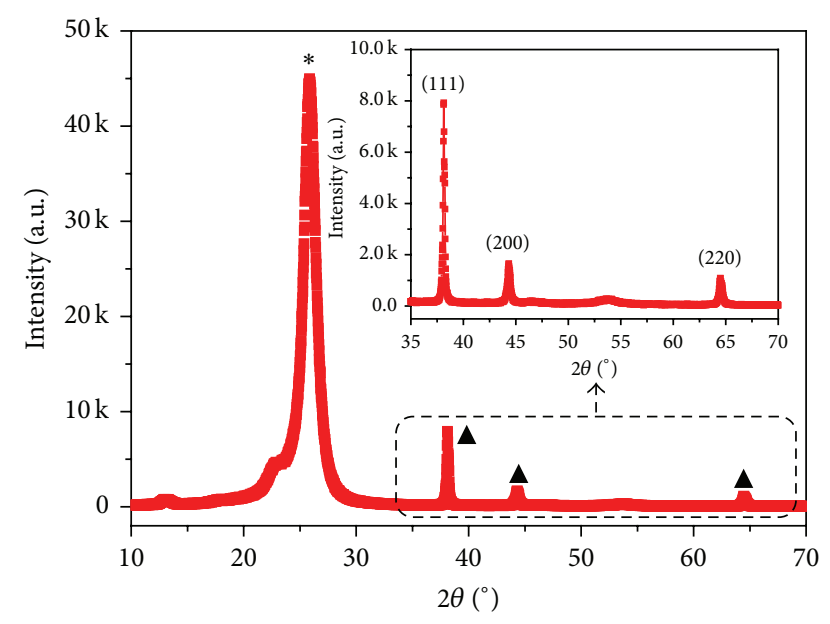

$\Delta \mathrm{Ag}$

* PET

FIGURE 4: XRD pattern of the PET and Ag nanoparticles. The asterisk “*” indicates the position of the PET substrate. The magnified view demonstrates the intensity of the different orientations of the crystalline lattice of Ag nanoparticles, as shown through XRD from different planes. for the Ag nanoparticles on the PET substrate. The XRD measurement further verifies that the peak intensity obtained for the Ag nanoparticles contains no lattice strain. Moreover, the particle size of Ag deposited on the PET film calculated by Scherrer's equation is $79.2 \mathrm{~nm}$. The tolerable discrepancy in the particle size of $50 \sim 130 \mathrm{~nm}$ generated from the SEM image is comparable to that obtained from the XRD measurement. During the deposition process, the original particles crashed into smaller grains, as confirmed by the XRD measurement, to form the flatter or homogeneous surface profile. It is observed that the metallization of the Ag nanoparticles on the PET substrate depends on the process and orientation of the metallization, as demonstrated by the XRD representation.

4.2. Device Characterization. The easiest and most effective way to investigate the impact of screen-printed Ag nanoparticles on PET film is by using atomic force microscopic (AFM) analysis. AFM images of the screen-printed Ag nanoparticles are shown in Figures 5(a) and 5(b). From analysis of the AFM measurement, we can conclude that the Ag nanoparticles are not completely homogenous over the surface of 


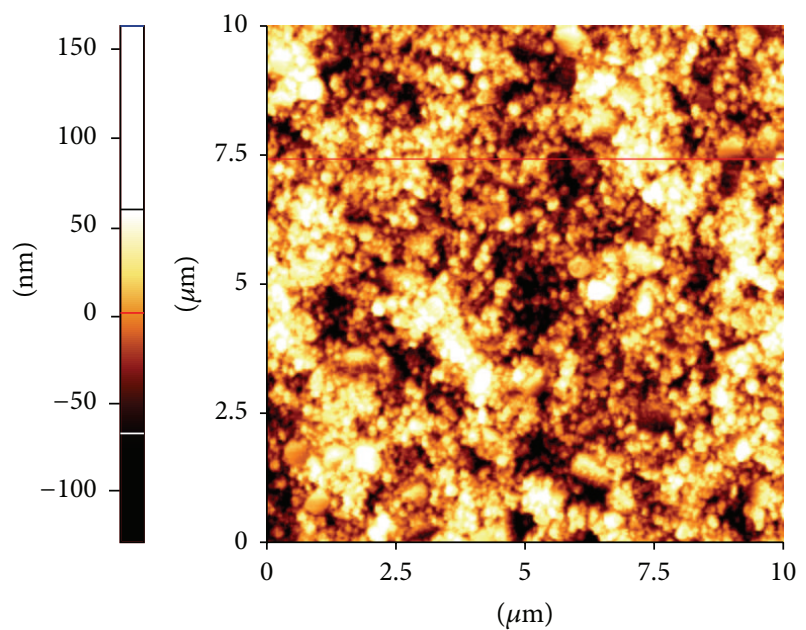

(a)

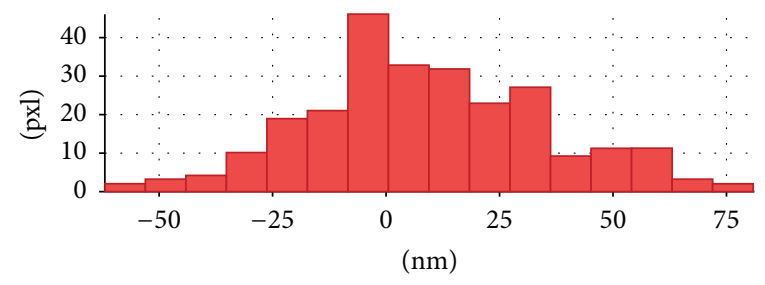

(c)

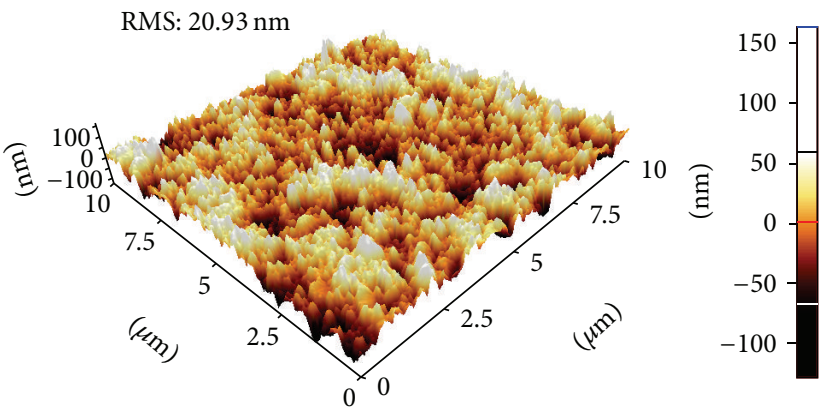

(b)

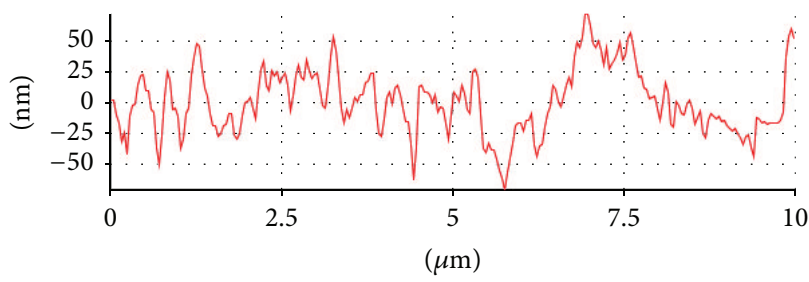

(d)

FIGURE 5: AFM images of Ag nanoparticles on the PET substrate: (a) two-dimensional, (b) three-dimensional, (c) histogram, and (d) lineprofile representations of the surface topology.

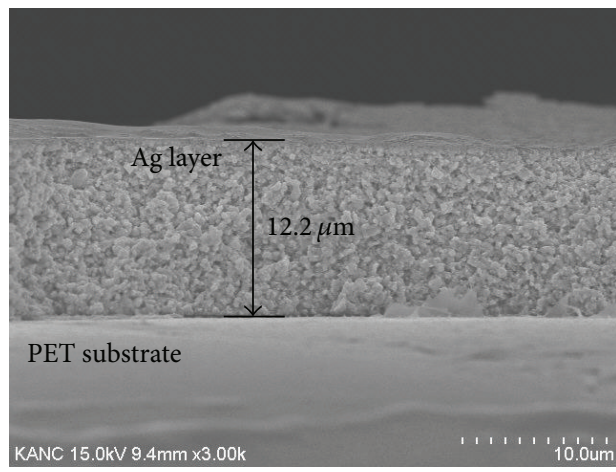

(a)

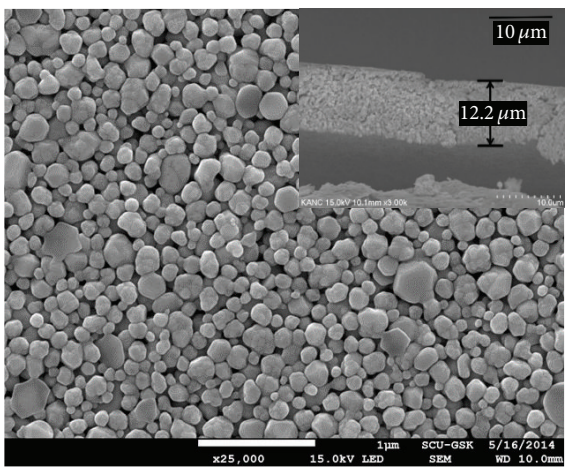

(b)

FIGURE 6: SEM images of screen-printed BSF representing the (a) thickness of the Ag layer with excellent adhesion to the PET substrate and relatively homogeneous distribution of the Ag nanoparticles and (b) the size of the Ag nanoparticles.

the substrate; rather, there exist some evenly distributed peaks and valleys. In other words, the surface of the screen-printed Ag nanoparticles appears comparatively flatter, representing an evenly distributed topographical structure [21]. The root mean square (RMS) value of $20.93 \mathrm{~nm}$ from its surface area of $108.82 \mu \mathrm{m}^{2}$ is sufficient for the simple and cost-effective screen-printing technique. In addition, the histogram and line graph also represent this surface topology with evenly distributed peaks and valleys. The maximum and minimum peaks and valleys created with screen-printing were approximately $50 \mathrm{~nm}$ peak-to-peak, as represented by Figures 5(c) and 5(d). We confirmed the Ag nanoparticle sizes using both SEM and transmission electron microscopy (TEM) images, which show a similar result. The size distribution of these particles was $50 \sim 130 \mathrm{~nm}$ with a thickness of $12.20 \mu \mathrm{m}$. Detailed information on the Ag nanoparticles is provided in Figures 6(a) and 6(b). The size of these nanoparticles does not significantly influenced the resistance unless they were less than $5 \mathrm{~nm}$. From printing with the proposed Ag ink, there was no considerable difference in the resistance. 


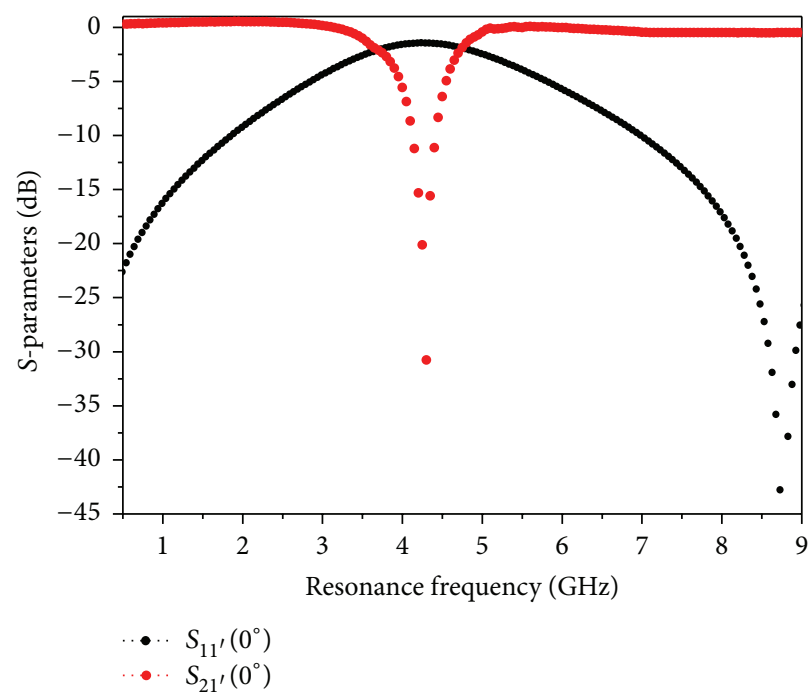

(a)

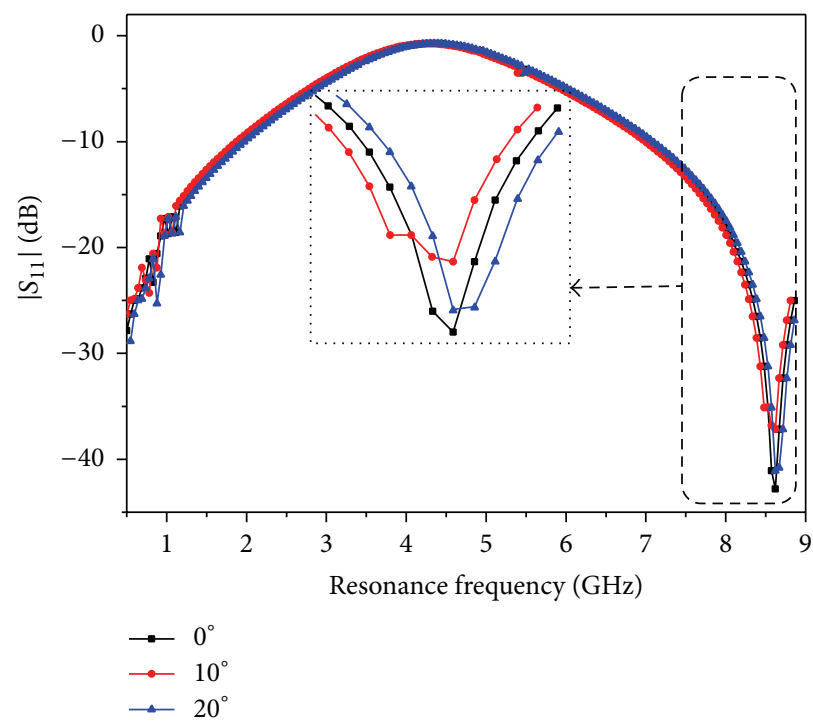

(c)

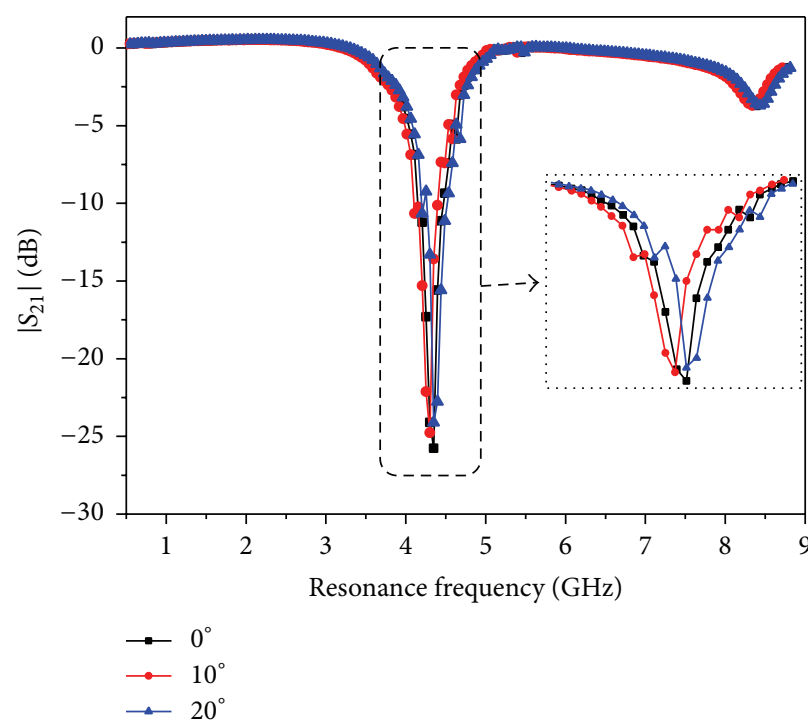

(b)

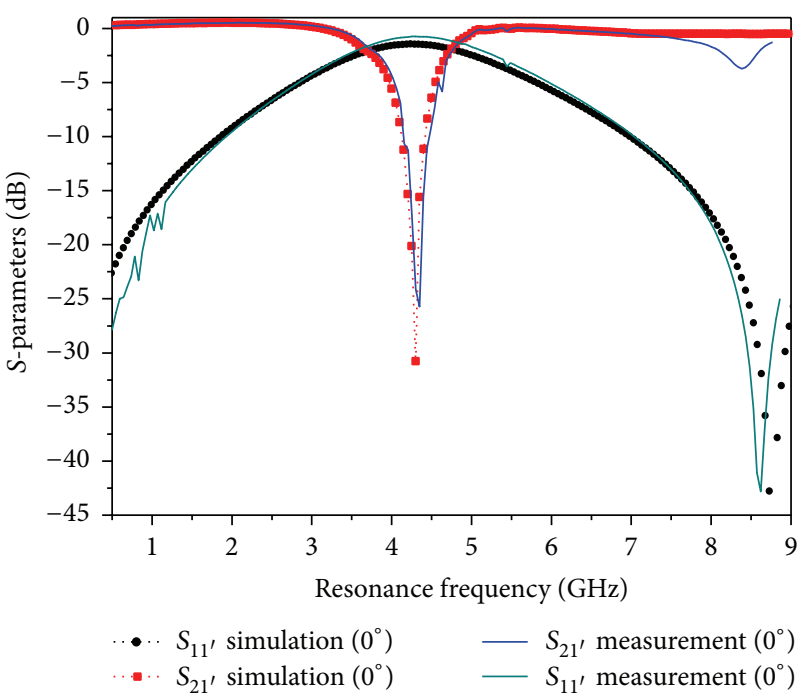

(d)

Figure 7: Measurement and simulation results of the flexible BSF on the PET substrate with respect to various bending angles. (a) Simulated $S$-parameters with a bending angle of $0^{\circ}$, (b) measured rejection depth $\left(S_{21}\right)$ with bending angles of $0^{\circ}, 10^{\circ}$, and $20^{\circ}$, (c) measured return loss $\left(S_{11}\right)$ with bending angles of $0^{\circ}, 10^{\circ}$, and $20^{\circ}$, and (d) comparative analysis of the simulated and measured results in normal conditions of the substrate orientation.

Good adhesion was obtained between the substrate and Ag nanoparticles, which verifies that the fabricated device is flexible and mechanically robust. Figure 6(a) clearly illustrates the cross-sectional view of the Ag metal layer with a thickness of $12.20 \mu \mathrm{m}$ with excellent adhesion with the PET substrate. Here, Figure 6(b) demonstrates that there is no aggregation among the particles with homogeneous surface profiles. The morphological characterization of the pattern suggests the RMS value of approximately $20.93 \mathrm{~nm}$. Additionally, the AFM analysis suggests that the peaks and valleys formed by the nanoparticles are homogeneous. This homogeneous distribution of particle size minimizes the roughness of the surface along with the radial loss of the signal at high frequency. This result is on account of the skin effect, as shown in supplementary Figure S2. The minimization of radial losses by maintaining the homogeneous surface profile helps to improve the return loss and attenuation depth of the signal $[22,23]$.

4.3. Radio Frequency Analysis. A flexible NBBS filter was designed and fabricated on the PET substrate with Ag nanoparticles as the conductive material for the transmission-line oriented at the normal condition $\left(0^{\circ}\right)$ to the surface, with the maximum signal attenuation level of $-25.88 \mathrm{~dB}$ and an attenuation pole at $4.35 \mathrm{GHz}$, as shown in Figures $7(\mathrm{a})$ and $7(\mathrm{~b})$. The device response for the various 
TABLE 1: Performance of the different measurements taken at various bending angles of the substrate.

\begin{tabular}{|c|c|c|c|c|c|c|c|c|c|}
\hline \multirow{2}{*}{$\mathrm{SN}^{\mathrm{a}}$} & \multirow{2}{*}{ Angle $^{\mathrm{b}}\left({ }^{\circ}\right)$} & \multicolumn{6}{|c|}{ Resonance frequency $(\mathrm{GHz})$} & \multirow{2}{*}{ Mean $\pm \operatorname{RSD}\left(f_{\mathrm{av}}^{\mathrm{d}}\right)$} & \multirow{2}{*}{$\operatorname{RSD}^{\mathrm{e}}(\%)$} \\
\hline & & 1st test & 2nd test & 3rd test & 4th test & 5th test & Mean $^{c}$ & & \\
\hline 1 & 0 & 4.35 & 4.35 & 4.35 & 4.34 & 4.35 & 4.34 & $4.34 \pm 0.10 \%$ & 0.10 \\
\hline 2 & 10 & 4.31 & 4.32 & 4.33 & 4.33 & 4.32 & 4.32 & $4.32 \pm 0.19 \%$ & 0.19 \\
\hline 3 & 20 & 4.34 & 4.35 & 4.33 & 4.34 & 4.32 & 4.34 & $4.34 \pm 0.26 \%$ & 0.26 \\
\hline
\end{tabular}

${ }^{\mathrm{a}} \mathrm{SN}$ denotes serial number.

${ }^{\mathrm{b}}$ Angle denotes the absolute value of the downward bending angle of the substrate.

${ }^{c}$ Mean denotes the average of the five experiments.

${ }^{\mathrm{d}} f_{\text {av }}$ denotes the final average resonance frequency.

${ }^{\mathrm{e}} \mathrm{RSD}$ denotes the relative standard deviation.

bending angles showed a small amount of deterioration of the performance parameters; however, this was considered within tolerable limits. The obtained results verify that the proposed device demonstrates consistent measurement results despite exposure to a different strain, as illustrated in supplementary Figure S3. For the device orientation of $10^{\circ}$ and $20^{\circ}$ to the horizontal surface, where $10^{\circ}$ and $20^{\circ}$ are the absolute values of the downward bending angle, it produced an attenuation level of $-24.79 \mathrm{~dB}$ and $-24.10 \mathrm{~dB}$ at a resonance frequency of $4.31 \mathrm{GHz}$ and $4.34 \mathrm{GHz}$, respectively, as shown in Figures $7(\mathrm{~b})$ and $7(\mathrm{c})$. The experimental data obtained from the first measurement of the multiple iterations has been considered for necessary illustration and analysis.

From the results of this experiment, we conclude that the device characteristics remain consistent despite its flexibility in various bending angles. The reproducibility of BSF was characterized by measuring the resonance frequency at various bending angles of the substrate. We carried out five different sets of experiments for each of the bending angles to observe the resonance frequency for each iteration of the experiment. Fifteen different experiments were performed in total. No device performance deterioration was observed over these numerous iterations of the measurement process. As a result, the relative standard deviation of less than $0.5 \%$ was observed for each bending angle of the substrate, as illustrated in Table 1 . These findings validate the reproducibility capability of the proposed flexible BSF.

In addition, a full-wave electromagnetic simulation of the filter by Sonnet is illustrated. It closely resembles the measurement result obtained from the VNA. A compact geometry was chosen for the compact size of an approximate range between $4 \mathrm{~mm}$ and $3.49 \mathrm{~mm}$. The maximum attenuation level of $-25.88 \mathrm{~dB}$ at the stopband exhibits a lower cutoff frequency of $4.18 \mathrm{GHz}$ and an upper cut-off frequency of $4.46 \mathrm{GHz}$ results, which results in an FBW $(-10 \mathrm{~dB})$ of $6.44 \%$. The stopband bandwidth and resonance frequency of the fabricated filter are closely matched with the simulated bandwidth and resonance frequency, as represented in Figure 7(d). The proposed method highlights a narrow stopband with sharp skirt selectivity, a higher rejection depth of $-25.88 \mathrm{~dB}$, and a lower return loss of $-0.75 \mathrm{~dB}$. The NBBS filter produced an excellent radio frequency (RF) phenomenon in the flexible PET substrate. It is therefore proved to be a suitable candidate for evolving technologies in the development of flexible radio devices and systems.

\section{Conclusion}

In this paper, we demonstrated the feasibility of realizing a bandstop filter at $4.35 \mathrm{GHz}$ through the injection of ink made from Ag nanoparticles on a commercially available PET substrate. This cost-effective screen-printing technology on a PET substrate is highly suitable for the realization of BSF filters with excellent in-and-out band performance at normal and bending conditions. The reproducibility of the device performance for various bending angles $\left(0^{\circ}, 10^{\circ}\right.$, and $\left.20^{\circ}\right)$ of the substrate was explored. The results showed an excellent RSD of less than $0.5 \%$, which confirms its flexibility as the BSF. Thus, the proposed printing method would facilitate the implementation of a simple, flexible, and reproducible bandstop filter with a homogeneous surface profile as a suitable candidate for C-band applications.

\section{Conflict of Interests}

The authors declare that there is no conflict of interests regarding the publication of this paper.

\section{Acknowledgments}

This research was supported by the Basic Science Research Program through the National Research Foundation of Korea (NRF) funded by the Ministry of Science, ICT and Future Planning (no. 2011-0030079) and a grant supported by the Korean Government (MEST) (no. 2012R1A1A2004366). This work was also supported by a Research Grant from Kwangwoon University in 2015. Additionally, the authors appreciate the financial support provided by the Ministry of Education in Korea through the Basic Science Research Program at Sunchon National University and by the Global Leading Technology Program of the Office of Strategic R\&D Planning (OSP) through the Ministry of Commerce, Industry and Energy, Republic of Korea (10042537, Printed Electronics Total Solution Development).

\section{References}

[1] Z. Wu, Y. Shim, and M. Rais-Zadeh, "Miniaturized UWB filters integrated with tunable notch filters using a silicon-based integrated passive device technology," IEEE Transactions on Microwave Theory and Techniques, vol. 60, no. 3, pp. 518-527, 2012. 
[2] C.-Y. Hsiao, S. S. H. Hsu, and D.-C. Chang, "A compact Vband bandpass filter in IPD technology," IEEE Microwave and Wireless Components Letters, vol. 21, no. 10, pp. 531-533, 2011.

[3] Y. Jung, H. Park, J.-A. Park et al., "Fully printed flexible and disposable wireless cyclic voltammetry tag," Scientific Reports, vol. 5, article 8105, 2015.

[4] H. Park, H. Kang, Y. Lee, Y. Park, J. Noh, and G. Cho, "Fully roll-to-roll gravure printed rectenna on plastic foils for wireless power transmission at $13.56 \mathrm{MHz}$," Nanotechnology, vol. 23, no. 34, Article ID 344006, 2012.

[5] J. Noh, M. Jung, K. Jung et al., "Fully gravure-printed D flipflop on plastic foils using single-walled carbon-nanotube-based TFTs," IEEE Electron Device Letters, vol. 32, no. 5, pp. 638-640, 2011.

[6] L.-L. Qu, D.-W. Li, J.-Q. Xue, W.-L. Zhai, J. S. Fossey, and Y.T. Long, "Batch fabrication of disposable screen printed SERS arrays," Lab on a Chip, vol. 12, no. 5, pp. 876-881, 2012.

[7] Y. Amin, Q. Chen, L. R. Zheng, and H. Tenhunen, "Development and analysis of flexible UHF antennas for green electronics," Progress in Electromagnetics Research, vol. 130, pp. $1-15,2012$.

[8] S. M. Bidoki, D. M. Lewis, M. Clark, A. Vakorov, P. A. Millner, and D. McGorman, "Ink-jet fabrication of electronic components," Journal of Micromechanics and Microengineering, vol. 17, no. 5, pp. 967-974, 2007.

[9] G. Kedawat, B. K. Gupta, P. Kumar et al., "Fabrication of a flexible UV band-pass filter using surface plasmon metalpolymer nanocomposite films for promising laser applications," ACS Applied Materials \& Interfaces, vol. 6, no. 11, pp. 8407-8414, 2014.

[10] V. K. Velidi, A. B. Guntupalli, and S. Sanyal, "Sharp-rejection ultra-wide bandstop filters," IEEE Microwave and Wireless Components Letters, vol. 19, no. 8, pp. 503-505, 2009.

[11] B. Shrestha and N. Y. Kim, "Microstrip wideband bandstop filter with open stubs for UWB applications," Microwave and Optical Technology Letters, vol. 57, no. 4, pp. 1003-1006, 2015.

[12] R. K. Maharjan, B. Shrestha, and N. Y. Kim, "Compact microstrip square open-loop bandpass filter using open stub," Electronics Letters, vol. 48, no. 6, pp. 333-334, 2012.

[13] N. Janković, R. Geschke, and V. Crnojević-Bengin, "Compact tri-band bandpass and bandstop filters based on Hilbert-fork resonators," IEEE Microwave and Wireless Components Letters, vol. 23, no. 6, pp. 282-284, 2013.

[14] E. J. Naglich, J. Lee, D. Peroulis, and W. J. Chappell, "A tunable bandpass-to-bandstop reconfigurable filter with independent bandwidths and tunable response shape," IEEE Transactions on Microwave Theory and Techniques, vol. 58, no. 12, pp.3770-3779, 2010.

[15] R. Dhakal and N.-Y. Kim, "A compact symmetric microstrip filter based on a rectangular meandered-line stepped impedance resonator with a triple-band bandstop response," The Scientific World Journal, vol. 2013, Article ID 457693, 7 pages, 2013.

[16] R. Dhakal and N.-Y. Kim, "A compact dual-band bandstop filter using a circular, folded, symmetric, meandered-line, steppedimpedance resonator," Microwave and Optical Technology Letters, vol. 56, no. 10, pp. 2298-2301, 2014.

[17] Y. Lan, Y. Xu, C. Wang et al., "X-band flexible bandpass filter based on ultra-thin liquid crystal polymer substrate," Electronics Letters, vol. 51, no. 4, pp. 345-347, 2015.

[18] Ł. Tymecki, E. Zwierkowska, and R. Koncki, "Screen-printed reference electrodes for potentiometric measurements," Analytica Chimica Acta, vol. 526, no. 1, pp. 3-11, 2004.
[19] J.-S. Kim, K.-B. Lee, J.-Y. Lee, H.-J. Lee, and H. Shin, "A 2.4GHz bandpass filter for flexible RF applications," Microwave and Optical Technology Letters, vol. 54, no. 2, pp. 384-387, 2012.

[20] R. Gomez-Garcia, M.-A. Sanchez-Soriano, K.-W. Tam, and Q. Xue, "Flexible filters: reconfigurable-bandwidth bandpass planar filters with ultralarge tuning ratio," IEEE Microwave Magazine, vol. 15, no. 5, pp. 43-54, 2014.

[21] K. Takano, T. Kawabata, C.-F. Hsieh et al., "Fabrication of terahertz planar metamaterials using a super-fine ink-jet printer," Applied Physics Express, vol. 3, no. 1, Article ID 016701, 2010.

[22] P. Waldow and I. Wolff, "The skin-effect at high frequencies," IEEE Transactions on Microwave Theory and Techniques, vol. 33, no. 10, pp. 1076-1082, 1985.

[23] X. Chen, "EM modeling of microstrip conductor losses including surface roughness effect," IEEE Microwave and Wireless Components Letters, vol. 17, no. 2, pp. 94-96, 2007. 

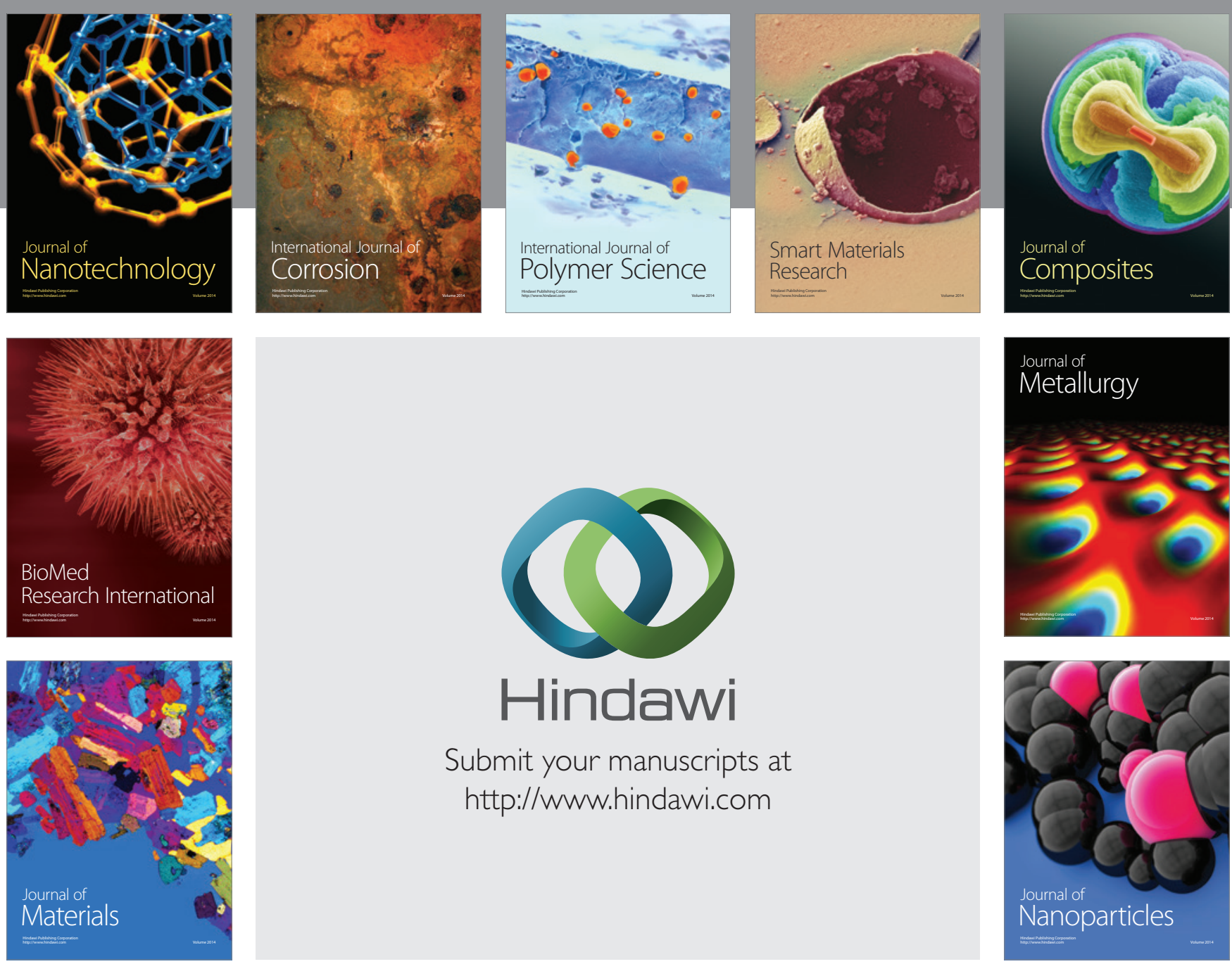

Submit your manuscripts at http://www.hindawi.com
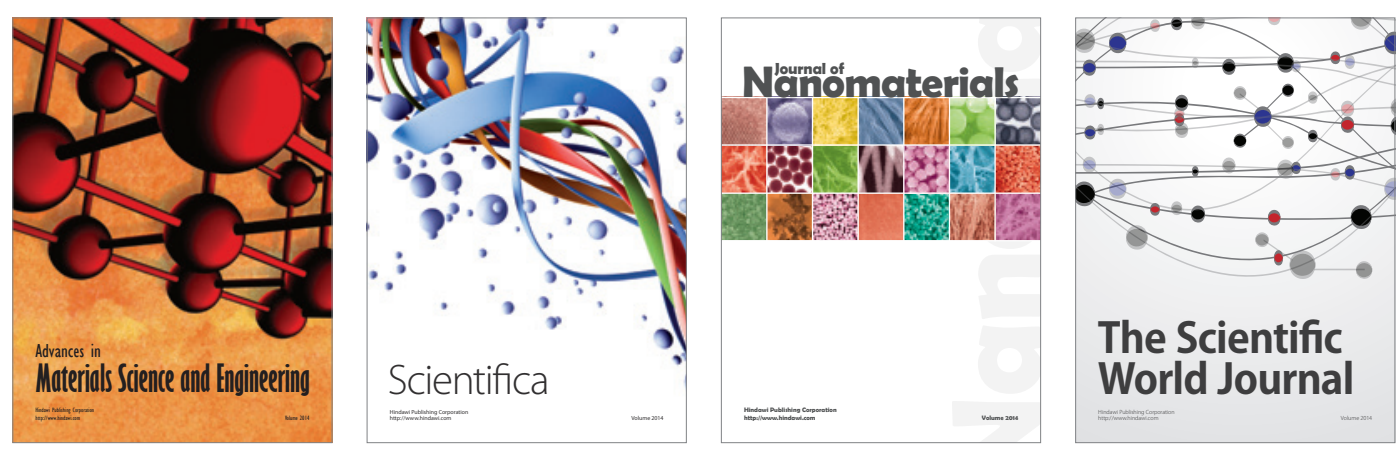

\section{The Scientific World Journal}
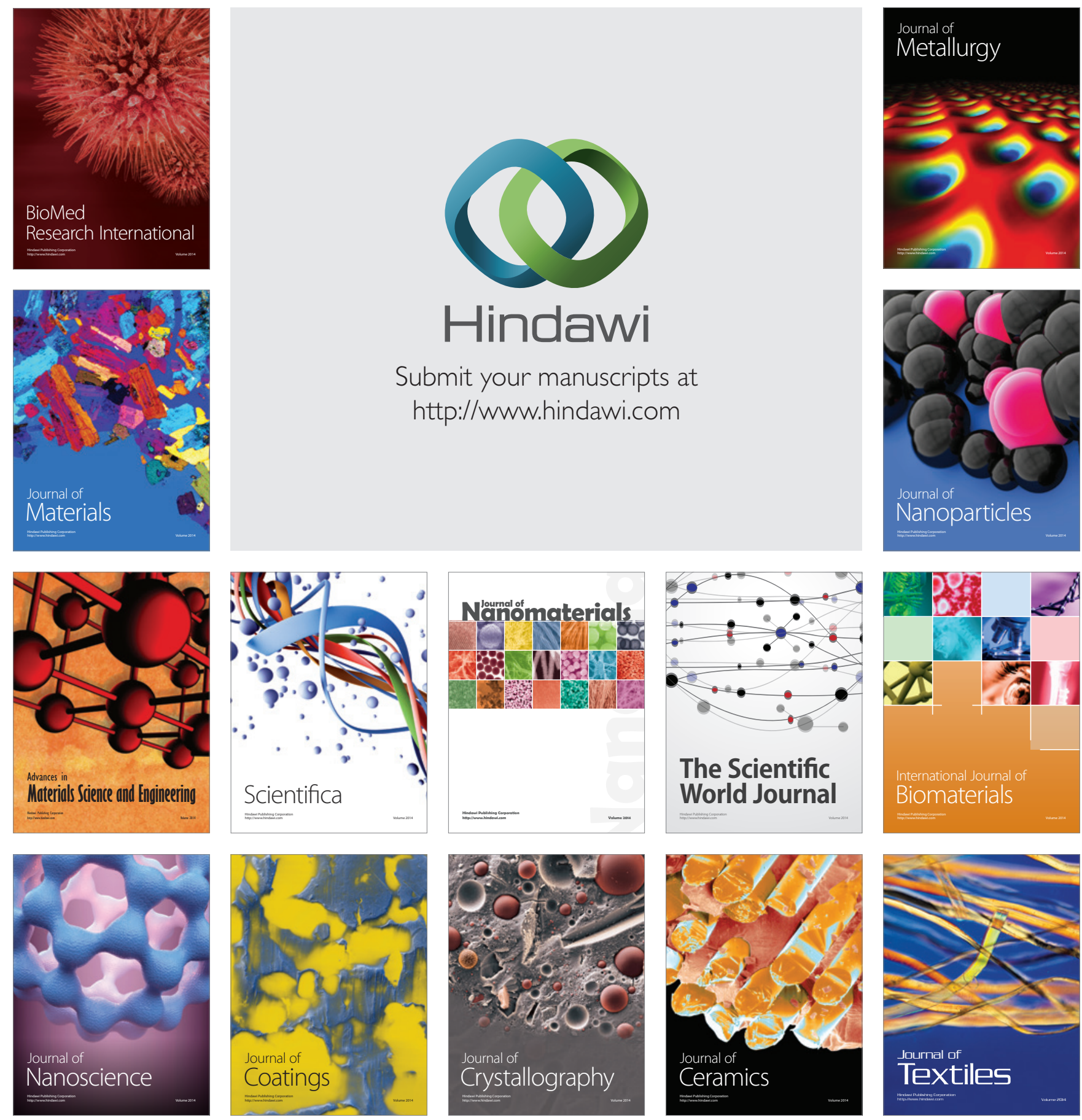